\title{
Imaging and Clinical Studies of the Choroid
}

\author{
Ying Liu ${ }^{1}$, Guoping Duan ${ }^{2, ~ * ~}$ \\ ${ }^{1}$ Department of Medical College, Hunan Provincial People's Hospital, The First Affiliated Hospital of Hunan Normal University, Changsha, \\ China \\ ${ }^{2}$ Department of Ophthalmology, Hunan Provincial People's Hospital, The First Affiliated Hospital of Hunan Normal University, Changsha, \\ China
}

\author{
Email address: \\ gniyuil1001@163.com (Ying Liu),dgp123004@sina.com (Guoping Duan) \\ ${ }^{*}$ Corresponding author
}

\section{To cite this article:}

Ying Liu, Guoping Duan. Imaging and Clinical Studies of the Choroid. International Journal of Ophthalmology \& Visual Science. Vol. 6, No. 2, 2021, pp. 101-107. doi: 10.11648/j.ijovs.20210602.17

Received: May 13, 2021; Accepted: May 25, 2021; Published: May 31, 2021

\begin{abstract}
The choroid, as an important nutrient tissue supplying the outer retinal layer and macular region, is primarily vascular in structure. The choroid accounts for approximately $70 \%$ of the blood flow to the entire uvea and, as the vascular system supplying the outer layer of the retina and the macula, it accounts for approximately $2 / 3$ of the blood flow to the entire eye. Because of its unique structure and function, the choroid plays a pivotal role in ocular disorders. For a long time, the study of the choroid has been in an exploratory stage due to its deep anatomical location and the limitations of the examination equipment. As research has progressed, it has become increasingly clear that choroid-related changes are a crucial factor in the pathogenesis of many ocular diseases. Qualitative changes in the choroid occur when ocular disorders occur, particularly when blood flow status is altered due to local or systemic disease, resulting in corresponding changes in choroidal blood flow, choroidal thickness, and choroidal volume. It has been shown that choroidal changes precede retinopathy in some eye diseases, for example, diabetic fundopathy: diabetic choroidopathy precedes diabetic retinopathy. In ocular disease, then, changes in choroidal structure and imaging play a prerequisite role in the early detection and treatment of the disease. This article, therefore, reviews the common clinical imaging modalities of the choroid in ophthalmology and the choroidal changes in related ocular diseases.
\end{abstract}

Keywords: Choroid, Imaging, Ophthalmology

\section{Introduction}

The choroid, a vital nutrient system for the outer layer of the retina and the central macular sulcus, relies heavily on its unique and productive blood-flowing vascular structure. A well-structured and functional choroidal tissue is essential for retinal function. Specifically, the primary function of the choroid is to supply nutrients and transport metabolites to the outer retinal layer, the retinal pigment epithelium, and the anterior optic nerve of the sieve plate; secondly, it is also the sole source of metabolic exchange in the central macular recess [1]. The choroidal vascular system is responsible for the nutrition and metabolism of the outer third of the retina, and when choroidal blood flow is abnormal, it leads to dysfunction and even apoptosis of photoreceptor cells in the retina [2]. It has been suggested that in diabetic retinopathy there is a tendency for structural changes in the choroid to occur early, meaning that the choroidal capillaries are altered before the retinal vasculature is altered and that such alterations may be an important mechanism in causing eye disease, meaning that the choroidal alterations may exist before the physician observes the retinal changes [3]. If other eye diseases are studied in more depth, the sequence of retinal and choroidal lesions can be explored as a theoretical basis for the early detection and treatment of clinical disease. Previous studies of the choroid have been limited for some time by the deep anatomical and histological location of the choroid, signal masking by pigment epithelium, signal absorption by water in the vitreous cavity, and abnormal signal interference, leading to a stagnation in the qualitative analysis of the choroid, with angiography and blood flow observation of the choroid being representative of these examinations. Technology has now allowed us to move forward with the quantitative and visual analysis of the 
choroid, including the measurement of Choroidal thickness and Choroidal volume. With the technical support of the new optical correlation tomography, clinically standardized values of choroidal structures are obtained during the quantitative measurement of the choroid, facilitating cross-referencing of findings by different investigators. Further findings summarise the sequence and characteristics of relevant structural tissue changes in ocular disease, providing new ideas for the development of clinical imaging and observational studies of ocular disease.

\section{Detection of the Choroid}

\subsection{Choroidal Blood Flow}

The choroid is mainly made up of blood vessels and normal blood flow in the eye is fed by two sets of non-interfering vessels: the central retinal vessels and the ciliary vessels. Of these, the posterior ciliary artery, once inside the eye, gives rise to two branches: one forms the short posterior ciliary artery: firstly, the Zinn ring, which supplies the optic papilla; secondly, it forms the posterior choroidal vessels (the capillaries of the choroid, the Sattler and Haller layers). The other branch forms the long posterior ciliary artery: it supplies the anterior uvea (which includes the iris, ciliary body, and anterior choroid).

Fundus angiography is the most widely used method for clinical observation of blood flow in ophthalmology. Fundus fluorescein angiography (FFA) and indocyanine green angiography (ICGA) are the two main types of fundus angiography. After blue light stimulation, the fluorescent material appears as a yellow-green image, and information about the time of visualization, the degree of filling of the vessels, and the presence of leakage can be used to infer the location, extent, and nature of the lesion in the fundus. However, observation of the choroid is particularly difficult due to factors such as rapid choroidal blood flow, retinal and choroidal pigment occlusion, and the molecular characteristics of sodium fluorescein, and is therefore now mainly used to observe the structure and blood flow of the retinal vascular circulation [4-5].

ICGA, a large-molecule fluorescent dye, was first applied to the cerebral circulation in dogs in 1969 by Kogure et al [6-7] and to the choroidal vascular circulation the following year. Flower [8-9] and others further investigated and improved indocyanine green angiography, which is a near-infrared light that is more penetrating and less absorbent in the blood than FFA, making ICGA more suitable than FFA in cases of nucleated cataracts, vitreous pooling, retinal hemorrhages and the appearance of abnormal blood vessels. Moreover, indocyanine green binds approximately $98 \%$ of plasma proteins $(80 \%$ of which bind to larger proteins: e.g. globulins and $\alpha 1$-lipoproteins) [10-11] and is less likely to penetrate choroidal vessels and leaks less in the choroid, thus providing a clearer and more visual indication of choroid-related structures and blood flow.

However, both of these methods of visualizing the intraocular vasculature are invasive, invasive and can cause a range of side effects such as nausea, vomiting [12], vagal reactions [13], and allergic reactions [14]. As a result, a non-invasive, rapid, and safe procedure has emerged: Optical Coherence Tomography Angiography (OCTA).

The principle of the OCTA examination is based on the element of motion in the eye: the blood flow - the movement of blood cells - and presents different levels of retinal vascular imaging according to the built-in software in the device, with different devices and their respective algorithms. It is also possible to combine the contrast produced by the moving blood cells with the surrounding static tissue through multiple B-scan scans of the same position and, after computer software, to generate the clinically required three-dimensional imaging graphics [15]; and it is possible to simultaneously select the retinal vessels to be observed at different levels, in addition to being able to show the deep retinal and choroidal vascular structures (analysis of the superficial vascular plexus, the deep vascular plexus, outer retinal layer, and choroidal capillaries). OCTA clearly shows vascular abnormalities such as enlarged central sulcus avascular areas, retinal avascular areas, microaneurysms, neovascularization of the retina and choroid, choroidal branch vessels, and choroidal polypoid lesions. However, the limitation of OCTA is that it is not yet possible to detect the filling time of fundus vessels and to observe the filling of vessels dynamically.

\subsection{Choroidal Thickness}

Choroidal thickness measurement refers to the thickness between the outer retinal pigment epithelium (RPE) and the junction between the choroid and the sclera [16]. The choroid is mainly composed of blood vessels and its thickness often varies considerably depending on the filling state of the vessels. According to a study by Goldenberg et al, the mean choroidal thickness (subfoveal choroidal thickness (SFCT)) of the central macular sulcus in healthy adults is $300 \mu \mathrm{m}$. Within $3 \mathrm{~mm}$ from the central recess, it is thickest above (approximately $308 \mu \mathrm{m}$ ), followed by the central recess (293 $\mu \mathrm{m})$, then temporal $(264 \mu \mathrm{m})$ and inferior $(263 \mu \mathrm{m})$, and thinnest on the nasal side $(174 \mu \mathrm{m})$ [17]. Meanwhile, choroidal thickness is influenced by various factors, such as age, gender, refractive error (eye axis), and diurnal pattern; and whether blood pressure (including diastolic and systolic blood pressure), intraocular pressure and perfusion pressure affect choroidal thickness is inconsistent with the findings of different scholars and needs to be further explored. Age and SFCT showed a negative correlation with age, i.e., the older the age, the smaller the choroidal thickness and the SFCT was significantly thicker in children under 10 years of age [18]. The SFCT is thicker in men than in women [19-20]. In the myopic refractive range greater than $-1.00 \mathrm{D}$, the SFCT decreased by $15 \mu \mathrm{m}$ for each $1 \mathrm{D}$ increase in myopic refraction and by $32 \mu \mathrm{m}$ for each $1 \mathrm{~mm}$ increase in the ocular axis [19]. Based on SFCT and $1.5 \mathrm{~mm}$ from the central recess, SFCT and nasal choroidal thickness were thicker in the evening than in the morning, while no significant difference was seen in 
temporal choroidal thickness [21].

The commonly used techniques such as FFA, ICGA, or OCTA can qualitatively analyze the choroidal vascular morphology and blood flow, but they cannot accurately present the choroidal thickness and its morphological features. The main tools available to quantify the structural features of the choroid are spectral-domain optical coherence tomography (SD-OCT) and swept-source optical coherence tomography (SS-OCT).

\subsubsection{Spectral-domain Optical Coherence Tomography}

SD-OCT consists of a broadband light source, a fiber optic Michelson interferometer, and a spectrometer system that accepts the interferometric signal [22]. Its imaging principle is based on coherent light imaging. The interference of coherent light results in different brightnesses depending on the phase relationship of the two beams. If the frequencies of the different light waves are slightly different, a new interference or beat frequency can be detected. Light interference using short coherence lengths produces a range of frequencies from different depths of tissue, depending on the depth of reflection. If the interference pattern is projected through the grating, the various frequency components will be dispersed. The closer the two path lengths match, the lower the frequencies, and the greater the path length mismatch for deeper structures, thus generating interference signals consisting of higher frequencies. These different signals and frequencies can be detected simultaneously, thus leading to imaging errors, which is where the limitations of SD-OCT imaging come into play. When using the Fourier transform, the location and intensity of the different reflections are determined by coding the frequencies. sd-OCT detects deep tissue, which produces higher frequency signals, but its detection method is nonlinear, so during detection, the higher frequency signals from deep tissue are mixed, producing more interference signals. Therefore, the sensitivity of SD-OCT decreases as the signal frequency increases, and the more the sensitivity decreases, the darker the imaging will be [23]. To better solve this problem, enhanced depth imaging optical coherence tomography (EDI-OCT) has been developed. EDI-OCT is the conversion of the interferometric signal into two conjugate images using the Fourier transform, which in practice usually shows one image, by adjusting the peak sensitivity to image the image deep in the posterior part of the eye, so that the choroidal structures appear [24].

\subsubsection{Swept-source Optical Coherence Tomography}

SS-OCT and SD-OCT use different light sources and different detection methods. SS-OCT separates different wavelengths of broad-spectrum light, receives the signal by using a balanced detector, and then reconstructs the information to obtain the signal returned at different depths, with much lower sensitivity attenuation than SD-OCT. The wavelength of the light wave of SS-OCT is longer than that of SD-OCT (in the range of $1 \mu \mathrm{m}$ ) so that this longer light wave can penetrate the tissue to a greater extent and achieve good choroidal tissue imaging. However, wavelengths beyond $1 \mu \mathrm{m}$ are attenuated by water absorption and imaging signal-to-noise is reduced [25-26]. In terms of imaging speed based on SS-OCT, SS-OCT achieves a faster sweep rate by separating the light source as described above and using a balanced detector. It has been shown that 3D stereoscopic imaging of the fundus with a larger field of view (approximately $80^{\circ}$ ) can be achieved using SS-OCT at 1.6 $\mathrm{MHz}$ [27]. In an analysis of choroidal structures in healthy eyes using SS-OCT compared to SD-OCT, Adhi [28] noted that SS-OCT was able to show $100 \%$ of the choroidal-scleral junction, which was a more significant advantage over EDI SD-OCT (73.6\%) and SD-OCT (68.4\%).

When measuring choroidal thickness, data from both measurement modalities showed inconsistencies, and Matsuo [29] et al. stated that SFCT assays obtained by both SD-OCT and SS-OCT instruments were reliable in normal subjects, with good reproducibility of each. However, the choroidal assay values measured with SS-OCT were somewhat larger than those measured with both SD-OCT instruments; therefore, the choroidal thickness should not be compared between SD-OCT and SS-OCT instruments. However, in the study by Pinilla [30] et al, the choroidal values measured by SD-OCT and SS-OCT instruments were also well reproducible, but the choroidal values obtained by SD-OCT were statistically higher than the values measured by SS-OCT. Therefore, based on the different data of choroidal values measured by SD-OCT and SS-OCT, it is proposed that the two measurement tools have different detection methods, that is, in addition to the effect caused by the different equipment, it is possible that both studies have a small sample size or whether they pose a certain geographical gap between different human populations. Therefore, each of them can be used for choroidal detection with good consistency and reproducibility of data, but the cross-referential value between them needs to be further investigation.

\subsection{Choroidal Volume}

Choroidal volume is the product of the choroidal thicknesses within a certain area, i.e. the choroidal volume. Therefore, all factors affecting choroidal thickness may affect choroidal volume, but the difference is that choroidal thickness, as a two-dimensional concept, responds to a longitudinal concept, whereas for choroidal volume, it is a three-dimensional structural response. As the lower choroidal boundary is wavy or curved, the use of choroidal thickness to describe the choroidal structure is a somewhat one-sided approach, and the choroidal volume within a region provides a more complete picture of the choroidal structure than the choroidal thickness value at a particular point or surface. Therefore, the author believes that in the future, as technology advances, choroidal volume testing may replace choroidal thickness testing. Similarly, there are two main methods for detecting choroidal volume: SD-OCT and SS-OCT, both of which can automatically analyze specific data on a choroidal volume using their algorithms and built-in software, enabling quantitative analysis of the structural characteristics of the choroid. 


\section{Choroidal Changes in Eye Disease}

With advances in the anatomy and imaging of the eye, knowledge of many ocular diseases has increased and many new ocular diseases have been recognized. The state of the choroid appears to be a key determinant in the pathogenesis of certain diseases, such as diabetic choroidopathy, age-related choroidal atrophy, chorioretinal inflammatory diseases, and ocular tumors.

\subsection{Diabetic Chorioretinopathy}

The incidence and prevalence of diabetes mellitus (DM) is increasing every year, and it is expected that by 2030, the total number of people aged 20-79 years with DM will be 578 million worldwide [31]. DM affects large and small blood vessels throughout the body, leading to vascular dysfunction and ultimately tissue degeneration and damage. In eye disease, the focus has been on retinopathy; in fact, some studies have illustrated that changes associated with choroidal vasculature are present before the retinal vasculature is diseased [3], known as diabetic choroidopathy (DC). Using scanning electron microscopy, Fryczkowski et al. showed that increased choroidal vascular curvature, focal vasodilation and narrowing, hypertrophy, vascular ring, and microaneurysm formation, "shedding" of choroidal capillaries, and the formation of areas of sinus-like structures between the equatorial choroidal lobules in patients with DM showed that changes in the choroidal capillaries resulting in reduced oxygenation of the outer retinal layers may contribute to diabetic retinopathy [3]. In addition, early pathological studies of DC have shown the following changes in the choroid: choroidal capillary loss, tortuosity, microaneurysms, deposits on Bruch's membrane choroidal neovascularisation, and have demonstrated that DC is an inflammatory disease [32]. It has been reported that the choroid thins in patients with early DR [33-35], but different studies have shown a trend towards thinning in the early stages and thickening in the later stages [36], and there are even some opinions that choroidal thickness shows a trend towards thickening in patients with DM [37-38]. The specific reasons for the inconsistent findings are unclear, and it is speculated that different ethnic differences, patients' systemic conditions, or medication use may be more likely to account for these differences.

\subsection{Central Serous Chorioretinopathy}

Central Serous Chorioretinopathy (CSC) is known to be one of the leading causes of vision loss in young people, with a prevalence of approximately 1 in 10,000 and a male to female ratio of between $72 \%$ and $88 \%$ [39]. CSC can present as an acute attack or as a chronic recurrence. In acute attacks, it usually resolves on its own within $2-3$ months, but in about $30 \%-50 \%$ of patients, it transforms into chronic CSC or recurrent acute CSC occurs, leading to retinal thinning or the appearance of subcentral macular fluid, which in turn leads to vision loss [40]. Secondly, CSC affects choroidal circulation disturbances, increases choroidal vascular permeability, and causes choroidal vasodilatation, leading to plasmacytoid pigment epithelial detachment and subretinal fluid formation, which is one of the reasons why it is called "chorioretinopathy" [41]. The increased choroidal thickness detected on SS-OCT confirms the role of the choroid in the pathogenesis of CSC $[42,43]$.

\subsection{Pathologic Myopia}

Progressive atrophy of the retinal pigment epithelium has been observed in patients with pathological myopia, and this change becomes more pronounced with increasing degrees of myopic macular degeneration (MMD). According to Ohno-Matsu et al., five types of myopic macular degeneration are defined: category 0: no myopic retinal degenerative lesions; category 1: tessellated fundus; category 2: diffuse retinal choroidal atrophy; category 3 : patchy retinal choroidal atrophy; and category 4: macular atrophy [44]. Significant obstruction of choroidal capillaries and progressive retinal degeneration have been observed in eyes with high myopia [45], with a reduced number of choroidal capillaries visible in the area of obstruction, and a similar reduction in choroidal thickness observed on SD-OCT [46]. Several studies have also found that reduced choroidal thickness correlates closely with the severity of MMD [47].

\subsection{Polypoidal Choroidal Vasculopaty}

In patients with polypoidal choroidal vasculopathy (PCV), SS-OCT-based studies have identified abnormally dilated choroidal vessels and compression of choroidal capillaries causing local ischemia, and these pathological changes may be relevant to the development of PCV [48]. Choroidal capillary atrophy and ischemia in patients with PCV allow for increased choroidal extravasation, which leads to increased choroidal permeability [49] and increased choroidal thickness. Several studies have shown that the ischaemic choroidal microenvironment promotes the expression of vascular endothelial growth factor (VEGF) and the production of neovascularization [50-51]. Therefore, changes in the choroid could be applied to the analysis of the efficacy of anti-VEGF drugs in subsequent studies.

\subsection{Age-Related Macular Degeneration}

In patients with Age-Related Macular Degeneration (AMD), the presence of choroidal neovascularization (CNV) often means that the disease has reached an advanced stage of AMD. The abnormal CNV breaks through the Bruch's membrane into the subretinal pigment epithelium (RPE) (type $1 \mathrm{CNV}$ ) or subretinal space (type $2 \mathrm{CNV}$ ) and, in some patients, anastomoses with the choroidal vascular system and manifests as a proliferating retinal haemangioma (type 3) [52] [53]. OCTA, based on SS-OCT imaging, can image CNV by semi-automatic segmentation of the outer retinal layer, subretinal, or below the RPE from a database, providing viable theoretical detection support for direct visualization of neovascular AMD and its microvascular details [54].

\subsection{Vogt-Koyanagi-Harada Syndrome}

Vogt-Koyanagi-Harada syndrome (VKH) is defined as 
bilateral chronic granulomatous uveitis with inflammation mainly involving the choroidal stroma, the RPE layer, and the outer retinal layer. ICGA showed choroidal vascular hyperfluorescence and delayed choroidal capillary circulation in the early stages of choroidal stromal inflammation, with later presentation as isofluorescence indicating inflammatory granuloma or persistent hypofluorescence indicating full choroidal granuloma [55]. On OCT, choroidal granulomas in VKH show a homogeneous rounded hyporeflective signal with well-defined margins and obscured signal beneath the granuloma [56]. SD-OCT and SS-OCT show increased CT in the acute phase of $\mathrm{VKH}$ and better choroidal resolution images with SS-OCT, especially in severe choroidal lesions $[39,57]$.

\section{Conclusion}

In summary, with continuous innovation in imaging technology, non-invasive, non-contact visualization of the choroid is now possible. Choroidal changes have been observed to play a significant role in the progression of disease in a growing number of ocular pathologies. It has been demonstrated that the overall structural features of the choroid can be observed in SS-OCT, so the changes in different tissues (mainly retina and choroid) and the connections between them in ocular diseases can be more deeply studied and explored. With further interest and research into the choroid, it is expected that future developments will allow for precise analysis of the layers of the choroid and exploration of the structural changes in the choroid in ocular disease.

\section{References}

[1] Hayreh SS. The blood supply of the optic nerve head and the evaluation of it - myth and reality $[\mathrm{J}]$. Prog Retin Eye Res, 2001, 20 (5): 563-593.

[2] Akkaya S. Spectrum of pachychoroid diseases [J]. Int Ophthalmol, 2018, 38 (5): 2239-2246.

[3] Fryczkowski AW, Hodes BL, Walker J. Diabetic choroidal and iris vasculature scanning electron microscopy findings $[\mathrm{J}]$. Int Ophthalmol. 1989, 13 (4): 269-279.

[4] Novotny HR, Alvis DL. A method of photographing fluorescence in circulating blood in the human retina $[\mathrm{J}]$. Circulation. 1961, 24: 82-86.

[5] Yoneya S, Noyori K. Improved visualization of the choroidal circulation with indocyanine green angiography $[\mathrm{J}]$. Arch Ophthalmol. 1993, 111 (9): 1165-1166.

[6] Kogure K, David NJ, Yamanouchi U, Choromokos E. Infrared absorption angiography of the fundus circulation [J]. Arch Ophthalmol. 1970, 83 (2): 209-214.

[7] Kogure K, Choromokos E. Infrared absorption angiography [J]. J Appl Physiol. 1969, 26 (1): 154-157.

[8] Flower RW, Hochheimer BF. Clinical infrared absorption angiography of the choroid [J]. Am J Ophthalmol. 1972, 73

\section{(3): $458-459$.}

[9] Flower RW. Extraction of choriocapillaris hemodynamic data from ICG fluorescence angiograms [J]. Invest Ophthalmol Vis Sci. 1993, 34 (9): 2720-2729.

[10] Baker KJ. Binding of sulfobromophthalein (BSP) sodium and indocyanine green (ICG) by plasma alpha-1 lipoproteins [J]. Proc Soc Exp Biol Med. 1966, 122 (4): 957-963.

[11] Cherrick GR, Stein SW, Leevy CM, Davidson CS. Indocyanine green: observations on its physical properties, plasma decay, and hepatic extraction [J]. J Clin Invest. 1960, 39 (4): 592-600.

[12] Lipson BK, Yannuzzi LA. Complications of intravenous fluorescein injections [J]. Int Ophthalmol Clin. 1989, 29 (3): 200-205.

[13] Pacurariu RI. Low incidence of side effects following intravenous fluorescein angiography $[\mathrm{J}]$. Ann Ophthalmol. 1982, 14 (1): 32-36.

[14] Stanga PE, Lim JI, Hamilton P. Indocyanine green angiography in chorioretinal diseases: indications and interpretation: an evidence-based update [J]. Ophthalmology. 2003, 110 (1): 15-23.

[15] Sambhav K, Grover S, Chalam KV. The application of optical coherence tomography angiography in retinal diseases $[\mathrm{J}]$. Surv Ophthalmol. 2017, 62 (6): 838-866.

[16] Nickla DL, Wallman J. The multifunctional choroid [J]. Prog Retin Eye Res. 2010, 29 (2): 144-168.

[17] Goldenberg D, Moisseiev E, Goldstein M, Loewenstein A, Barak A. Enhanced depth imaging optical coherence tomography: choroidal thickness and correlations with age, refractive error, and axial length [J]. Ophthalmic Surg Lasers Imaging. 2012, 43 (4): 296-301.

[18] Fujiwara A, Shiragami C, Shirakata Y, Manabe S, Izumibata S, Shiraga F. Enhanced depth imaging spectral-domain optical coherence tomography of subfoveal choroidal thickness in normal Japanese eyes [J]. Jpn J Ophthalmol. 2012, 56 (3): 230-235.

[19] Li XQ, Larsen M, Munch IC. Subfoveal choroidal thickness in relation to sex and axial length in 93 Danish university students [J]. Invest Ophthalmol Vis Sci. 2011, 52 (11): 8438-8441.

[20] Wei WB, Xu L, Jonas JB, et al. Subfoveal choroidal thickness: the Beijing Eye Study [J]. Ophthalmology. 2013, 120 (1): 175-180.

[21] Toyokawa N, Kimura H, Fukomoto A, Kuroda S. Difference in morning and evening choroidal thickness in Japanese subjects with no chorioretinal disease [J]. Ophthalmic Surg Lasers Imaging. 2012, 43 (2): 109-114.

[22] Izatt JA, Hee MR, Owen GM, Swanson EA, Fujimoto JG. Optical coherence microscopy in scattering media [J]. Opt Lett. 1994, 19 (8): 590-592.

[23] Mrejen S, Spaide RF. Optical coherence tomography: imaging of the choroid and beyond [J]. Surv Ophthalmol. 2013, 58 (5): 387-429.

[24] Spaide RF, Koizumi H, Pozzoni MC. Enhanced depth imaging spectral-domain optical coherence tomography $[\mathrm{J}]$. Am J Ophthalmol. 2008; 146 (4): 496-500. 
[25] Jacques SL. Optical properties of biological tissues: a review [J]. Phys Med Biol. 2013, 58 (11): R37-R61.

[26] Marschall S, Klein T, Wieser W, et al. Fourier domain mode-locked swept source at $1050 \mathrm{~nm}$ based on a tapered amplifier [J]. Opt Express. 2010, 18 (15): 15820-15831.

[27] Klein T, Wieser W, Reznicek L, Neubauer A, Kampik A, Huber R. Multi-MHz retinal OCT. Biomed Opt Express. 2013; 4 (10): 1890-1908.

[28] Adhi M, Liu JJ, Qavi AH, et al. Choroidal analysis in healthy eyes using swept-source optical coherence tomography compared to spectral domain optical coherence tomography [J]. Am J Ophthalmol. 2014, 157 (6): 1272-1281.e1.

[29] Matsuo Y, Sakamoto T, Yamashita T, Tomita M, Shirasawa M, Terasaki H. Comparisons of choroidal thickness of normal eyes obtained by two different spectral-domain OCT instruments and one swept-source OCT instrument [J]. Invest Ophthalmol Vis Sci. 2013, 54 (12): 7630-7636.

[30] Pinilla I, Sanchez-Cano A, Insa G, Bartolomé I, Perdices L, Orduna-Hospital E. Choroidal Differences between Spectral and Swept-source Domain Technologies [J]. Curr Eye Res. 2021, 46 (2): 239-247.

[31] Saeedi P, Petersohn I, Salpea P, et al. Global and regional diabetes prevalence estimates for 2019 and projections for 2030 and 2045: Results from the International Diabetes Federation Diabetes Atlas, 9th edition [J]. Diabetes Res Clin Pract, 2019, 157: 107843.

[32] Lutty GA. Diabetic choroidopathy [J]. Vision Res, 2017, 139: 161-167.

[33] Vujosevic S, Martini F, Cavarzeran F, Pilotto E, Midena E. Macular and peripapillary choroidal thickness in diabetic patients [J]. Retina. 2012; 32 (9): 1781-1790.

[34] Querques G, Lattanzio R, Querques L, et al. Enhanced depth imaging optical coherence tomography in type 2 diabetes [J]. Invest Ophthalmol Vis Sci, 2012, 53 (10): 6017-6024.

[35] Regatieri CV, Branchini L, Carmody J, Fujimoto JG, Duker JS Choroidal thickness in patients with diabetic retinopathy analyzed by spectral-domain optical coherence tomography [J]. Retina, 2012, 32 (3): 563-568.

[36] Kase S, Endo H, Yokoi M, et al. Choroidal thickness in diabetic retinopathy in relation to long-term systemic treatments for diabetes mellitus [J]. Eur J Ophthalmol, 2016, 26 (2): 158-162.

[37] Rewbury R, Want A, Varughese R, Chong V. Subfoveal choroidal thickness in patients with diabetic retinopathy and diabetic macular oedema [J]. Eye (Lond), 2016, 30 (12): 1568-1572.

[38] Melancia D, Vicente A, Cunha JP, Abegão Pinto L, Ferreira J. Diabetic choroidopathy: a review of the current literature [J]. Graefes Arch Clin Exp Ophthalmol, 2016, 254 (8): 1453-1461.

[39] Pichi F, Carrai P, Ciardella A, Behar-Cohen F, Nucci P. Comparison of two mineralcorticosteroids receptor antagonists for the treatment of central serous chorioretinopathy [J]. Int Ophthalmol, 2017, 37 (5): 1115-1125.

[40] Nicholson B, Noble J, Forooghian F, Meyerle C. Central serous chorioretinopathy: update on pathophysiology and treatment [J]. Surv Ophthalmo, 2013, 58 (2): 103-126.

[41] Chhablani J, Pichi F, Silva R, et al. Antiangiogenics in choroidal neovascularization associated with laser in central serous chorioretinopathy [J]. Retina, 2016, 36 (5): 901-908.

[42] Kuroda S, Ikuno Y, Yasuno Y, et al. Choroidal thickness in central serous chorioretinopathy [J]. Retina, 2013, 33 (2): 302-308.

[43] Jirarattanasopa P, Ooto S, Tsujikawa A, et al. Assessment of macular choroidal thickness by optical coherence tomography and angiographic changes in central serous chorioretinopathy [J]. Ophthalmology, 2012, 119 (8): 1666-1678.

[44] Ohno-Matsui K, Kawasaki R, Jonas JB, et al. International photographic classification and grading system for myopic maculopathy [J]. Am J Ophthalmol, 2015, 159 (5): 877-83.e7.

[45] Okabe S, Matsuo N, Okamoto S, Kataoka H. Electron microscopic studies on retinochoroidal atrophy in the human eye [J]. Acta Med Okayama, 1982, 36 (1): 11-21.

[46] Cheung CM, Loh BK, Li X, et al. Choroidal thickness and risk characteristics of eyes with myopic choroidal neovascularization $[\mathrm{J}]$. Acta Ophthalmol, 2013, 91 (7): e580-e581.

[47] Wong CW, Phua V, Lee SY, Wong TY, Cheung CM. Is Choroidal or Scleral Thickness Related to Myopic Macular Degeneration? [J]. Invest Ophthalmol Vis Sci, 2017, 58 (2): 907-913.

[48] Dansingani KK, Balaratnasingam C, Naysan J, Freund KB. En face imaging of pachychoroid spectrum disorders with swept-source optical coherence tomography [J]. Retina, 2016, 36 (3): 499-516.

[49] Pang CE, Freund KB. Pachychoroid neovasculopathy [J]. Retina, 2015, 35 (1): 1-9.

[50] Dansingani KK, Gal-Or O, Sadda SR, Yannuzzi LA, Freund KB. Understanding aneurysmal type 1 neovascularization (polypoidal choroidal vasculopathy): a lesson in the taxonomy of 'expanded spectra' - a review [J]. Clin Exp Ophthalmol, 2018, 46 (2): 189-200.

[51] Dansingani KK, Balaratnasingam C, Klufas MA, Sarraf D, Freund KB. Optical Coherence Tomography Angiography of Shallow Irregular Pigment Epithelial Detachments In Pachychoroid Spectrum Disease [J]. Am J Ophthalmol, 2015, 160 (6): 1243-1254.e2.

[52] Ambati J, Ambati BK, Yoo SH, Ianchulev S, Adamis AP. Age-related macular degeneration: etiology, pathogenesis, and therapeutic strategies [J]. Surv Ophthalmol, 2003, 48 (3): 257-293.

[53] Yannuzzi LA, Negrão S, Iida T, et al. Retinal angiomatous proliferation in age-related macular degeneration $[\mathrm{J}]$. Retina, 2001, 21 (5): 416-434.

[54] Kuehlewein L, Bansal M, Lenis TL, et al. Optical Coherence Tomography Angiography of Type 1 Neovascularization in Age-Related Macular Degeneration [J]. Am J Ophthalmol, 2015, 160 (4): 739-48.e2.

[55] Herbort CP, Mantovani A, Bouchenaki N. Indocyanine green angiography in Vogt-Koyanagi-Harada disease: angiographic signs and utility in patient follow-up [J]. Int Ophthalmol, 2007, 27 (2-3): 173-182. 
[56] Chee SP, Chan SN, Jap A. Comparison of Enhanced Depth Imaging and Swept Source Optical Coherence Tomography in Assessment of Choroidal Thickness in Vogt-Koyanagi-Harada Disease [J]. Ocul Immunol Inflamm, 2017, 25 (4): 528-532.
[57] Silpa-Archa S, Ittharat W, Chotcomwongse P, Preble JM, Foster CS. Analysis of Three-Dimensional Choroidal Volume with Enhanced Depth Imaging Findings in Patients with Recurrent Vogt-Koyanagi-Harada Disease [J]. Curr Eye Res, 2020, 1-8. 